\title{
Effects of High-Intensity Resistance Training and Aerobic Exercise on Expression of ABCG4, ABCG5 and ABCG8 Genes in Female Athletes
}

Reza Bagheri (PhD)

Department of Exercise Physiology,

University of Isfahan, Isfahan, Iran

Samaneh Darroudi (MSc)

Department of Exercise Physiology,

Ferdowsi University of Mashhad,

Mashhad, Iran

Seyed Mojtaba Hosseini (PhD)

Department of Physical Education and

Sports Sciences, Islamic Azad

University, Neyshabur Branch,

Neyshabur, Iran

Hossein Nikkar (PhD)

Young Researchers and Elite Club,

Neyshabur Branch, Islamic Azad

University, Neyshabur, Iran

Fatemeh Khodadadi (MSc)

Department of Exercise Physiology,

Ferdowsi University of Mashhad,

Mashhad, Iran

Somaye Kasraee (MSc)

Department of Exercise Physiology,

Ferdowsi University of Mashhad,

Mashhad, Iran

Mahboube Sazegar (PhD)

Department of Physical Education and

Sports Sciences, Islamic Azad

University, Neyshabur Branch,

Neyshabur, Iran

Amir Rashidlamir (PhD)

Department of Exercise Physiology,

Ferdowsi University of Mashhad,

Mashhad, Iran

Corresponding author: Amir

Rashidlamir

Email: Rashidlamir@um.ac.ir

Tel: +989151514174

Address: Department of Exercise

Physiology, Ferdowsi University of

Mashhad, Mashhad, Iran

Received: 2019/05/15

Revised: 2019/06/9

Accepted: 2019/07/28

(c) (i) (8)

This work is licensed under a Creative

Commons Attribution 4.0 License.

\begin{abstract}
Background and objectives: Atherosclerotic cardiovascular disease is the leading cause of death in industrialized countries. High level of plasma lipids including cholesterol and triglycerides is one of the most important risk factors of atherosclerosis. Previous studies have shown that three members of the ATP-binding cassette, subfamily G (ABCG4, ABCG5 and ABCG8) are involved in transporting sterols across membranes. The purpose of this study was to assess the effect of high-intensity resistance training (HIRT) and aerobic exercise (AE) on expression of the $\mathrm{ABCG}, \mathrm{ABC} 5$ and $\mathrm{ABC} 00$ genes in female athletes.

Methods: Twenty-four female athletes from the Khorasan Province (Iran) were randomly selected and assigned into three groups: control $(\mathrm{N}=0), \mathrm{AE}(\mathrm{N}=0)$ and HIRT $(\mathrm{N}=0)$. The subjects in the HIRT group and the AE group performed exercise at intensity of 75-80\% of 1-repetion maximum and 75-80\% of maximum heart rate, respectively. Blood samples were collected at baseline and immediately after the exercise session. After isolation of lymphocytes by centrifugation and purification of mRNA, gene expression changes were investigated by Real-Time-PCR. Data were analyzed using one-way ANOVA and the Tukey's test.
\end{abstract}

Results: Both training protocols significantly increased the expression of ABCG4, ABCG5 and $\mathrm{ABC} \mathrm{C} 8 \mathrm{in}$ the subjects $(\mathrm{P}<0.05)$, and there was no significant difference in the expression of these genes between the experimental groups $(\mathrm{P}>0.05$.)

Conclusion: A single session of HIRT and AE may have beneficial effects on prevention of atherosclerosis .

Keywords: Aerobic Exercise, High Intensity Resistance Training, ABCG4, ABCG5, ABCG0, PBWN.

This paper should be cited as: Bagheri R, Darroudi S, Hosseini SM, Nikkar H, Khodadadi F, Kasraee S, Sazegar M, Rashidlamir A. [Effects of High-Intensity Resistance Training and Aerobic Exercise on Expression of ABCG4, ABCG5 and ABCG8 Genes in Female Athletes]. mljgoums. 2020; 14(3): 40-45. 


\section{INTRODUCTION}

The most frequent cause of death in industrialized countries is atherosclerosis, which is closely linked with plasma lipoprotein levels and subsequently cholesterol deposition in vascular cells (1). Cholesterol and phospholipids are imperative for the body; however, excessive accumulation of cholesterol or fat is toxic and a risk factor for atherosclerosis (2). Reverse cholesterol transport (RCT) is a process in which excess cholesterol from peripheral tissues, including arterial wall macrophages, is returned to the liver, where it is broken and excreted. This process prevents cholesterol adherence to the artery wall (3). Reduction of cholesterol intake in intestines and transport of excess cholesterol in peripheral tissues to the liver and biliary cholesterol secretion are necessary for maintaining cholesterol homeostasis. Excessive cholesterol in the liver is excreted directly to the bile or indirectly to bile acids (4).

ATP-binding cassette transporters (ABC) have an important role in RCT and are divided into seven groups of $A$ to $G$ (5). Regarding the important role of $\mathrm{ABCG}$ and $\mathrm{ABCA}$ transporters in the absorption and elimination of sterols, the role of these genes in determining the genetic significance of lipid levels is currently under consideration (6). These two members play a role in lipid transfer and provide a protective mechanism for removal of excess cholesterol in the body. However, ABCA1 and ABCG1 are not involved in transfer of cholesterol from the liver to bile ducts (7). ATP-binding cassette transporters consist of a large family of membrane proteins that utilize ATP hydrolysis energy for transporting various molecules across the biological membrane (8). As a member of the $\mathrm{ABC}$ family and a lipid carrier, ABCG have several members including ABCG1, ABCG2, ABCG3, ABCG4, ABCG5, ABCG8, ABCG11, ABCG12 and ABCG26 (9). The $A B C$ protein is involved in the RCT process and the metabolism of plasma and tissue cholesterol (10). In fact, all ABCGs except for G2, play an important role in the RCT process (11).

Studies have shown that ABCG1, ABCG4, ABCG5 and ABCG8 are the major determinant of on high-density lipoprotein (HDL) levels and the most important protective agents against atherosclerosis (12).
Moreover, ABCG4, ABCG5 and ABCG8 are key regulators in the removal of cholesterol and phospholipid from macrophage foam cells. In addition, the expression of these regulatory factors, especially in the leucocyte, is important for homeostatic regulation (13).

In recent years, various studies have been conducted on the effect of exercise on the ABC-family proteins. Connolly et al. showed that a single bout of intensive exercise changes the expression of hundreds of genes, including inflammatory and pro-inflammatory genes as well as genes necessary for physiological functions in healthy men (14). In addition, a number of studies have shown that genetic factors may contribute to the improvement of cardiovascular risk factors in response to regular physical activity (15). Büttner et al. examined the effect of treadmill running at $80 \% \quad \mathrm{VO}_{2} \max$ and $60 \% \quad \mathrm{VO}_{2} \max$ on gene expression profile of white blood cell and reported upregulation of 450 genes and downregulation of 150 genes in male participants (16). Previous studies have shown that expression of the $A B C G 4, A B C G 5$ and $A B C G 8$ genes is influenced by various factors, .including a high-fat diet and physical training Although several studies have investigated the effect of different types of training on expression of $A B C A 1$ and $A B C G 1(17,18)$, no study has yet investigated the response of $A B C G 4, A B C G 5$ and $A B C G 8$ to exercise. It has been suggested that a high cholesterol diet can increase ABCG5/ABCG8 levels and cholesterol transfer from the liver to the biliary duct (19). The aim of the present study was to investigate effects of aerobic exercise (AE) and high-intensity resistance training (HIRT) on the expression of $A B C G 4, A B C G 5$ and $A B C G 8$ genes in lymphocytes of female athletes.

\section{MATERIALS AND METHODS}

Overall, 24 female athletes with mean age of 21.48 years and mean body mass index of $22.28 \mathrm{~kg} \cdot \mathrm{m}^{2}$ were selected for participation in the study. Exclusion criteria included having cardiovascular disease, diabetes, hypertension or other health risk factors and consumption of alcohol or sport supplements. Informed consent was obtained from all participants prior to participation in the study(20).

First, the participants were familiarized with the study procedures and were randomly assigned into three groups:_aerobic exercise 
(AE; $\mathrm{n}=8$ ), high-intensity resistance training (HIRT; $\mathrm{n}=8$ ) and control $(\mathrm{C} ; \mathrm{n}=8)$. Measurements were performed at baseline and after eight weeks at the same time of day. The participants were instructed not to alter their regular lifestyle and dietary habits during the study.

The subject were weighed on emptied bladder using a digital scale (lumbar, China) to the nearest $0.1 \mathrm{~kg}$. Height was measured with a stadiometer (Race industrialization, China) to the nearest $0.1 \mathrm{~cm}$. Body fat percentage was determined using a caliper device (Lafayette, model 01127 , USA) with an accuracy of $1 \mathrm{~mm}$. Three skinfold sites were measured, namely above the iliac, femoral neck and tricepsbrachii on the right side. Then, body fat percentage was calculated using the Jackson and Pollock formula (21).

To minimize dietary variability, the participants were required to submit 3-day (two weekdays and one weekend) food records at baseline and eight weeks after the training session. Each dietary item was entered into the Diet Analysis Plus software (version 10, Cengage, USA) and total energy consumption as well as the amount of energy derived from proteins, fats and carbohydrates were assessed. Maximal strength testing took place 24 hours after the body composition measurement. The participants warmed up for 10 minutes and rested for five minutes between every three attempts. They were instructed to refrain from food intake for two hours before the testing; however, water consumption was allowed. The test was carried out every two weeks. The maximal strength was measured using the following formula (22):

1 repetition maximum $(1 \mathrm{RM})=$ weight $/$ (1.0278-0.0278×reps)

Each set of HIRT included nine exercises at nine stations, with 20 repetitions per exercise, and no rest interval between stations. Three sets with three minutes rest intervals between sets were performed. The intensity of the exercises was set at $75-80 \%$ of $1 \mathrm{RM}$. The total time of the training sessions was 40 minutes, consisting of 15 minutes of warm up, 10 minutes of cool down and 15 minutes of training. The control group did not perform any exercise. The exercises included chest press, leg press, seated rowing, leg extension, biceps curl and standing calf raise.

In the HIRT group, subjects ran for 1.5 mile at 75 to $80 \%$ of maximum heart rate (HR). In the $\mathrm{AE}$ group, subjects ran on treadmill for 1.5 miles at $70 \%$ of maximum HR. The HR was monitored using a HR monitor.

Blood samples were taken after 12 hours of overnight fasting. The samples were collected in tubes containing EDTA anticoagulant and were transferred to the laboratory. A semiquantitative RT-PCR method was used to perform the mRNA purification test. The lymphocytes were put in liquid nitrogen and completely crushed by mortal and pestle. The degraded tissue was homogenized in the RLT buffer. The use of the rotor-stator homogenizer provides more levels of RNA. Tissue powder and liquid nitrogen were poured into a $2 \mathrm{ml}$ RNase free tube and allowed the nitrogen to be evaporated, but the lymphocytes were not frozen. An adequate amount of RLT buffer was added. Lysate was transferred directly to the QIAshredder column located on the tube and centrifuged for 2 minutes and high speed. Total RNA was extracted from 80 to $100 \mathrm{mg}$ of tissue using an RNA purification kit (AccuZol, Bioneer, Cat. No: k3090, Korea). Complementary DNA (cDNA) was produced using oligo- $(\mathrm{dt})_{18}$ primers $(0.25 \mu \mathrm{g}$ per reaction) and a cDNA synthesis kit (AccuPower RT PreMix) according to the manufacturer's instructions. Real-time PCR was performed using the LightCycler apparatus (Corbet, Australia). Real-time quantitative PCR was performed using QuantiFast SYBR Green PCR Kit (Cat. No. 204052; Qiagen, $\mathrm{GmbH}$, Germany) in a $15 \mu \mathrm{L}$ reaction solution containing $0.5 \mu \mathrm{L}$ singlestrand cDNA, $7.5 \mu \mathrm{L}$ Master Mix, $1 \mu \mathrm{L}$ of the each primers $(5 \mathrm{pmol} / \mu \mathrm{L})$ and $5 \mu \mathrm{L} \mathrm{dH} 2 \mathrm{O}$. Sequences of the primers used in the experiment are listed in table1.

Table 1. The sequences of the primers used in the study

\begin{tabular}{crc}
\hline Gene & Forward primer & Reverse primer \\
\hline ABCG4 & 5'-CCGAGACCAGCCGCTTC-'3 & '5-TCCCAAAGACTGGGCAACTAAG-'3 \\
\hline ABCG5 & '-CGTCAGATTTCCAATGACTTCCG-3'5 & '-TCCGTCCTCCAGTTCATAGTACA-3'5 \\
\hline ABCG8 & -AGGCTCAGTTACAGGCTCAGAG-3'5 & -GTCCCACTTCTGCTGGCATGAT-3'5 \\
\hline Actin & AGCCTTCCTTCCTGGGCATGG & AGCACTGTGTTGGCGTACAGGTC \\
\hline
\end{tabular}


Results of the PCR experiment were analyzed by gel electrophoresis.

Normality of data and homogeneity of variance between groups were investigated using the Shapiro-Wilk test and the Levene's test, respectively. Parametric tests were used to examine intergroup variations. One-way ANOVA and Tukey's test were used to detect significant differences. All statistical analyses were performed in IBM SPSS Statistics 24.0 at significance of 0.05 .

\section{RESULTS}

Baseline anthropometric characteristics of the participants are presented in table2. A single bout of $\mathrm{AE}$ and HIRT increased the expression of $A B C G 4, A B C G 5$ and $A B C G 8$ (Table 3). The results of the Tukey's test indicated no significant difference in the expression of the mentioned genes between the two training groups $(\mathrm{P}>0.05)$. Moreover, the anthropometric variables did not change significantly after the training intervention.

Table 2. Baseline anthropometric characteristics of the participants

\begin{tabular}{|c|c|c|c|c|}
\hline Variable & Control & HIRT & $\mathbf{A E}$ & P-value \\
\hline Weight $(\mathrm{kg})$ & $59.6 \pm 7.6$ & $56.3 \pm 9.3$ & $58.2 \pm 7.8$ & 0.42 \\
\hline Body fat percentage $(\%)$ & $19.6 \pm 1.6$ & $18.2 \pm 3.1$ & $19.6 \pm 5.5$ & 0.77 \\
\hline
\end{tabular}

Table 3. Changes in expression of $A B C G 4, A B C G 5$ and $A B C G 8$ genes in each study group

\begin{tabular}{|c|c|c|c|c|c|c|}
\hline Gene & Stage & Control & HIRT & $\mathbf{A E}$ & $\mathbf{F}$ & P-value \\
\hline \multirow[t]{2}{*}{ ABCG4 } & Pre-training & $0.37 \pm 0.45$ & $0.2 \pm 0.15$ & $0.69 \pm 0.45$ & 19.91 & 0.001 \\
\hline & Post-training & $0.25 \pm 0.23$ & $2.32 \pm 0.58$ & $2.74 \pm 0.66$ & & \\
\hline \multirow[t]{2}{*}{ ABCG5 } & Pre-training & $0.41 \pm 0.33$ & $0.54 \pm 0.34$ & $0.64 \pm 0.49$ & 9.21 & 0.001 \\
\hline & Post-training & $0.26 \pm 0.25$ & $1.22 \pm 0.45$ & $1.37 \pm 0.64$ & & \\
\hline \multirow[t]{2}{*}{ ABCG8 } & Pre-training & $0.32 \pm 0.21$ & $0.44 \pm 0.41$ & $0.7 \pm 0.37$ & 7.21 & 0.004 \\
\hline & Post-training & $0.32 \pm 0.24$ & $1.33 \pm 0.52$ & $1.58 \pm 0.91$ & & \\
\hline
\end{tabular}

\section{DISCUSSION}

A single bout of both AE and HIRT increased the expression of $A B C G 4, A B C G 5$ and $A B C G 8$ in lymphocytes of female athletes. As mentioned previously, $A B C G 4, A B C G 5$ and $A B C G 8$ play an important role in the RCT process. ABCG5 has been thought to reduce biliary cholesterol secretion and absorption of dietary cholesterol (23). ABCG4, which has the same structure and function as ABCG1, transfers cholesterol from macrophages to the liver and regulates cholesterol homeostasis in the brain (24). ABCG1 and ABCG4 increase cholesterol efflux to HDL. Numerous studies have shown that physical activity can increase the expression of $A B C A 1$ (2, 16, 18, 25). Despite the known effects of physical activity on $\mathrm{ABC}$ family gene expression, few studies have evaluated the effects of different types of exercise on expression of these genes, especially genes other than ABCA1 $(13,16)$. Wang et al. reported that ABCG1 and ABCG4 stimulate isotopic cholesterol efflux to HDL-2 and HDL-3. They also observed that the combination of ABCG1 and ABCG4 resulted in a slight increase in the cholesterol efflux (26).

Research has shown that physical activity followed by dietary intervention may affect expression of $A B C G 4, A B C G 5$ and $A B C G 8$ in lymphocyte, the liver, small intestine and heart of mice. GhanbariNiaki et al. reported that eight weeks of endurance training $(25 \mathrm{~m} / \mathrm{min}, 60$ minutes per session, five days a week) led to expression of $A B C G 4, A B C G 5$ and $A B C G 8$ genes in the small intestine and kidney of mice (18).

Another study showed that $A B C G 4$ expression changes were associated with significant variation in plasma HDL-cholesterol level in the liver and intestinal tissues (10).

A fat-free diet or consumption of unsaturated fatty acids increase the expression of $A B C A 1, A B C G 4$, $A B C G 5$ and $A B C G 8$, while low and moderate intensity exercise can also increase the oxidation of fatty acids (27).

Recently, Côté et al. showed that six weeks of advanced exercise training $(16-26 \mathrm{~m} / \mathrm{min}$ at $0-10 \%$ 
slope, 15-60 $\mathrm{min} /$ day, five times a week) increase the expression of $A B C G 5$ and $A B C G 8$ genes in the small intestine of mice, while an atherogenic diet (high fat / high cholesterol) had no such effect (28). In a study by Rashidlamir et al., an aerobic or resistance training session significantly increased the expression of $A B C G 1$ (29). Previous studies demonstrated that short-term endurance training at moderate intensity increases expression of the $\mathrm{ABC}$ genes, pre-beta HDL level and eventually HDL, all of which promote RCT and prevent atherosclerosis (30). It has been suggested that $A B C A 1, A B C G 1$, $A B C G 4, A B C G 5$ and $A B C G 8$ expression may be due to increased lipoprotein lipase, liver lipase, beta-HDL and lecithin-cholesterol acyltransferase, which are important factors in the prevention of cardiovascular disease (2).

\section{REFERENCES}

1.Rashidlamir A, Saadatnia A. The Effect of Eight Weeks of Wrestling and Wrestling Technique Based Circuit Trainingon Lymphocyte ABCA1 Gene Expression and Plasma Apolipoprotein AI. World journal of sport sciences. 2011; 4(2): 144-150.

2. Schmitz G, Kaminski WE, Orsó E. ABC transporters in cellular lipid trafficking. Current opinion in lipidology. 2000;11(5):493-501.

3. Yancey PG, Bortnick AE, Kellner-Weibel G, De la LleraMoya M, Phillips MC, Rothblat GH. Importance of different pathways of cellular cholesterol efflux. Arteriosclerosis, thrombosis, and vascular biology. 2003;23(5):712-9.

4. Miller G, Miller N. Plasma-high-density-lipoprotein concentration and development of ischaemic heart-disease. The lancet. 1975;305(7897):16-9.

5. Woodward OM, Köttgen A, Köttgen M. ABCG transporters and disease. The FEBS journal. 2011; 278(18): 3215-25. doi: 10.1111/j.1742-4658.2011.08171.x

6. Tanisawa K, Tanaka M, Higuchi M. Gene-exercise interactions in the development of cardiometabolic diseases. The Journal of Physical Fitness and Sports Medicine. 2016;5(1):25-36.

7. Fitzgerald ML, Mujawar Z, Tamehiro N. ABC transporters, atherosclerosis and inflammation. Atherosclerosis. 2010; 211(2): 361-70. doi: 10.1016/j.atherosclerosis.2010.01.011.

8. Davidson AL, Chen J. ATP-binding cassette transporters in bacteria. Annual review of biochemistry. 2004;73(1):241-68.

9. Choi H, Jin JY, Choi S, Hwang JU, Kim YY, Suh MC, et al. An ABCG/WBC-type ABC transporter is essential for transport of sporopollenin precursors for exine formation in developing pollen. The Plant Journal. 2011;65(2):181-93.

10. Ghanbari-Niaki A, Rahmati-Ahmadabad S. Effects of a fixed-intensity of endurance training and pistacia atlantica supplementation on ATP-binding cassette G4 expression. Chinese medicine. 2013; 8(1): 23. doi: 10.1186/1749-8546-8-23.

11. Kusuhara H, Sugiyama Y. ATP-binding cassette, subfamily $G$ (ABCG family). Pflügers Archiv-European Journal of Physiology. 2007;453(5):735-44.

12. Durstine JL, Grandjean PW, Davis PG, Ferguson MA, Alderson NL, DuBose KD. Blood lipid and lipoprotein adaptations to exercise. Sports medicine. 2001;31(15):1033-62. DOI: 10.2165/00007256-200131150-00002.
In the present study, we did not evaluate the effect of the trainings on nuclear receptors that are present in the cholesterol outflow, namely peroxisome proliferator-activated receptors, liver $\mathrm{X}$ receptor and farnesoid $\mathrm{X}$ receptor that are thought to be involved in regulation of $A B C G 4, A B C G 5$ and $A B C G 8$ expression (28), which is a limitation of our study .

\section{CONCLUSION}

Our results demonstrate that AE and HIRT are beneficial for cardiovascular health. Therefore, we recommend athletes, especially females, to perform HIRT in addition to AE to achieve greater physiological benefits.

\section{ACKNOWLEDGEMENTS}

We would like to thank all participants for their cooperation in the present study.

\section{CONFLICT OF INTEREST}

The authors declare no conflict of interest.

13. Shulenin S, Schriml L, Remaley A, Fojo S, Brewer B, Allikmets R, et al. An ATP-binding cassette gene (ABCG5) from the $A B C G$ (White) gene subfamily maps to human chromosome $2 p 21$ in the region of the Sitosterolemia locus. Cytogenetic and Genome Research. 2001;92(3-4):204-8.

14. Connolly PH, Caiozzo VJ, Zaldivar F, Nemet D, Larson J, Hung S-p, et al. Effects of exercise on gene expression in human peripheral blood mononuclear cells. Journal of applied physiology. 2004;97(4):1461-9.

15. Hoang A, Tefft C, Duffy SJ, Formosa M, Henstridge DC, Kingwell BA, et al. ABCA1 expression in humans is associated with physical activity and alcohol consumption. Atherosclerosis. 2008; 197(1): 197-203.

16. Büttner P, Mosig S, Lechtermann A, Funke H, Mooren FC. Exercise affects the gene expression profiles of human white blood cells. Journal of applied physiology. 2007; 102(1): 26-36.

17. Butcher LR, Thomas A, Backx K, Roberts A, Webb R, Morris K. Low-intensity exercise exerts beneficial effects on plasma lipids via PPARgamma. Med Sci Sports Exerc. 2008; 40(7): 1263-70. doi: 10.1249/MSS.0b013e31816c091d.

18. Ghanbari-Niaki A, Khabazian BM, Hossaini-Kakhak SA, Rahbarizadeh F, Hedayati M. Treadmill exercise enhances $A B \backslash A 1$ expression in rat liver. Biochemical and biophysical research communications. 2007;361(4):841-6.

19. Bagheri R, Rashidlamir A, Ashtary-Larky D, Wong A, Alipour M, Motevalli MS, et al. Does Green Tea Extract Enhance the Anti-inflammatory Effects of Exercise on Fat Loss? Br J Clin Pharmacol. 2019. doi: 10.1111/bcp.14176.

20. Matsuura F, Oku H, Koseki M, Sandoval JC, Yuasa-Kawase $\mathrm{M}$, Tsubakio-Yamamoto $\mathrm{K}$, et al. Adiponectin accelerates reverse cholesterol transport by increasing high density lipoprotein assembly in the liver. Biochemical and biophysical research communications. 2007; 358(4): 1091-5. DOI: 10.1016/j.bbrc.2007.05.040.

21. Bagheri R, Moghadam BH, Church DD, Tinsley GM, Eskandari M, Moghadam $\mathrm{BH}$, et al. The effects of concurrent training order on body composition and serum concentrations of follistatin, myostatin and GDF11 in sarcopenic elderly men. Exp Gerontol. 2020; 133: $110869 . \quad$ doi: 10.1016/j.exger.2020.110869.

22. Ghanbari-Niaki A, Saghebjoo M, Rashid-Lamir A, Fathi R, Kraemer RR. Acute circuit-resistance exercise increases expression of lymphocyte agouti-related protein in young women. Experimental Biology and Medicine. 2010; 235(3): 32634. 
23. Graf GA, Cohen JC, Hobbs HH. Missense mutations in $A B C G 5$ and $A B C G 8$ disrupt heterodimerization and trafficking. Journal of Biological Chemistry. 2004; 279(23): 24881-8.

24. Tarr PT, Tarling EJ, Bojanic DD, Edwards PA, Baldán Á. Emerging new paradigms for ABCG transporters. Biochim Biophys Acta. 2009;1791(7):584-93. doi: 10.1016/j.bbalip.2009.01.007.

25. Tofighi A, Rahmani F, Qarakhanlou BJ, Babaei S. The effect of regular aerobic exercise on reverse cholesterol transport A1 and apo lipoprotein aI gene expression in inactive women. Iranian Red Crescent Medical Journal. 2015; 17(4): e26321. doi: 10.5812/ircmj.17(4)2015.26321.

26. Wang N, Lan D, Chen W, Matsuura F, Tall AR. ATP. binding cassette transporters $G 1$ and $G 4$ mediate cellular cholesterol efflux to high-density lipoproteins. Proceedings of the National academy of Sciences of the United States of America. 2004; 101(26): 9774-9.
27. Gelissen IC, Harris M, Rye K-A, Quinn C, Brown AJ, Kockx M, et al. ABCA1 and ABCG1 synergize to mediate cholesterol export to apoA-I. Arteriosclerosis, thrombosis, and vascular biology. 2006; 26(3): 534-40.

28. Côté I, Sock ETN, Lévy É, Lavoie J-M. An atherogenic diet decreases liver FXR gene expression and causes severe hepatic steatosis and hepatic cholesterol accumulation: effect of endurance training. European journal of nutrition. 2013; 52(5): 1523-32.

29. Rashidlamir A. Investigation of the Effect of Aerobic and Resistance Exercises on Peripheral Blood Mononuclear Cells ABCG1 Gene Expression in Female Athletes. SSU_Journals. 2012; 20(1): 1-9.

30. Khabazian BM, Ghanbari-Niaki A, Safarzadeh-Golpordesari A, Ebrahimi M, Rahbarizadeh F, Abednazari H. Endurance training enhances ABCA1 expression in rat small intestine. European journal of applied physiology. 2009;107(3):351-8. 